По мнению рецензентов и редколлегии сборника, в публикуемой статье Л.Е. Захарова некоторые положения являются спорными, а оценка автором степени новизны концепции несколько преувеличенной. Тем не менее редколлегия считает, что публикация статьи полезна как материал для размышления и обсуждения, и приглашает читателей высказать своё мнение по существу статьи.

УДК 621.039.623.634:546.34

\title{
LiWALL FUSION - THE NEW CONCEPT OF MAGNETIC FUSION
}

\author{
L.E. Zakharov (Princeton University, Princeton Plasma Physics Laboratory, MS-27, P.O. Box 451, Princeton, \\ New Jersey 08543)
}

It relies on innovative plasma regimes with low edge density and high temperature. The approach combines fueling the plasma by neutral injection beams with the best possible elimination of outside neutral gas sources, which may cool down the plasma edge. Prevention of cooling the plasma edge suppresses the dominant, temperature gradient related turbulence in the core. Such an approach is much more suitable for controlled fusion than the present practice, relying on high heating power for compensating essentially unlimited turbulent energy losses.

Key words: fusion power, tokamaks, magnetic confinement, burning plasma.

ТЕРМОЯДЕРНЫЙ СИНТЕЗ С ЛИТИЕВОЙ СТЕНКОЙ - НОВАЯ КОНЦЕПЦИЯ ТЕРМОЯДЕРНЫХ УСТАНОВОК С МАГНИТНЫМ УДЕРЖАНИЕМ ПЛАЗМЫ. Л.Е. ЗАХАРОВ. Использование выдающейся способности слоёв жидкого лития откачивать изотопы водорода открывает новый подход к термоядерному синтезу с магнитным удержанием плазмы, называемый термоядерный синтез с литиевой стенкой. Он основан на новых режимах удержания, характеризующихся низкой плотностью и высокой температурой на границе плазмы. Этот подход сочетает инжекцию пучков быстрых атомов топлива в плазму с максимально возможным подавлением внешних источников нейтрального газа, который может охладить пограничную плазму. Предотвращение охлаждения края плазмы подавляет турбулентность в плазменном шнуре, связанную с температурным градиентом. Такой подход значительно более рационален для управляемого термоядерного синтеза, чем современные подходы, ориентирующиеся на большую мощность нагрева плазмы для компенсации по существу неограниченных турбулентных потерь энергии.

Ключевые слова: термоядерная энергия, токамаки, магнитное удержание плазмы, термоядерная реакция в плазме.

\section{INTRODUCTION}

In the 1990-s, TFTR [1] and JET [2] experiments have demonstrated the controlled fusion power of 10 and 16 MW correspondingly. Still the target value $\mathrm{Q}^{\mathrm{DT}}=1$ of a fusion power factor was not achieved, indicating the limitation of the adopted approach to fusion, relying on enhancement in the size of the plasma, magnetic field, plasma current, and heating power.

At the same time, new physics was introduced in TFTR experiments, related to Li conditioning of the belt limiter. This was achieved by $\mathrm{Li}$ pellet injection during conditioning shots, which preceded the high performance TFTR supershots. At the end of the program the DOLLOP technique for creating Li aerosol was used for the wall conditioning. Li conditioning dramatically improved plasma performance and was used routinely for TFTR supershots.

After termination of the TFTR program, the experiments on T-11M [3] demonstrated outstanding abilities of lithium surface to pump out plasma particles. This result has initiated a new understanding of the effect of lithium on the tokamak plasma.

On Dec. 23, 1998, I telephoned Sergei Krasheninnikov with a simple question: "What happens if the inner walls of tokamak are covered with lithium which absorbs all the particles from the plasma". His answer was instantaneous: "The plasma temperature profile will be flat with edge temperature as high as the core temperature". In addition to a lithium pumping surface, Sergei emphasized the necessity of core fueling. Although core fueling seemed to be very problematic, my reaction was "Then, the fusion problem is solved". In 1998, this was an intuition, which has been grown to a self-consistent concept [4-6], called the LiWall Fusion (LiWF).

This paper describes the basics of LiWF. The essence of LiWF is very simple: core plasma fueling by Neutral Beam Injection (NBI), and plasma particle pumping by a liquid lithium layer. LiWF intends to implement the 
fundamental understanding that in approaching fusion conditions it is much more efficient to prevent the plasma from cooling by neutrals coming from the walls, rather than rely, as in the conventional approach, on extensive heating power in order to compensate the turbulent energy losses, which are essentially unlimited.

The conventional approach to fusion is illustrated in Fig. 1, where the large recycling at the walls cools down the plasma edge, causes the peaked temperature profile, while keeping the density flat because of the dominantly near edge particle source. The plasma temperature is low compared to the beam energy.
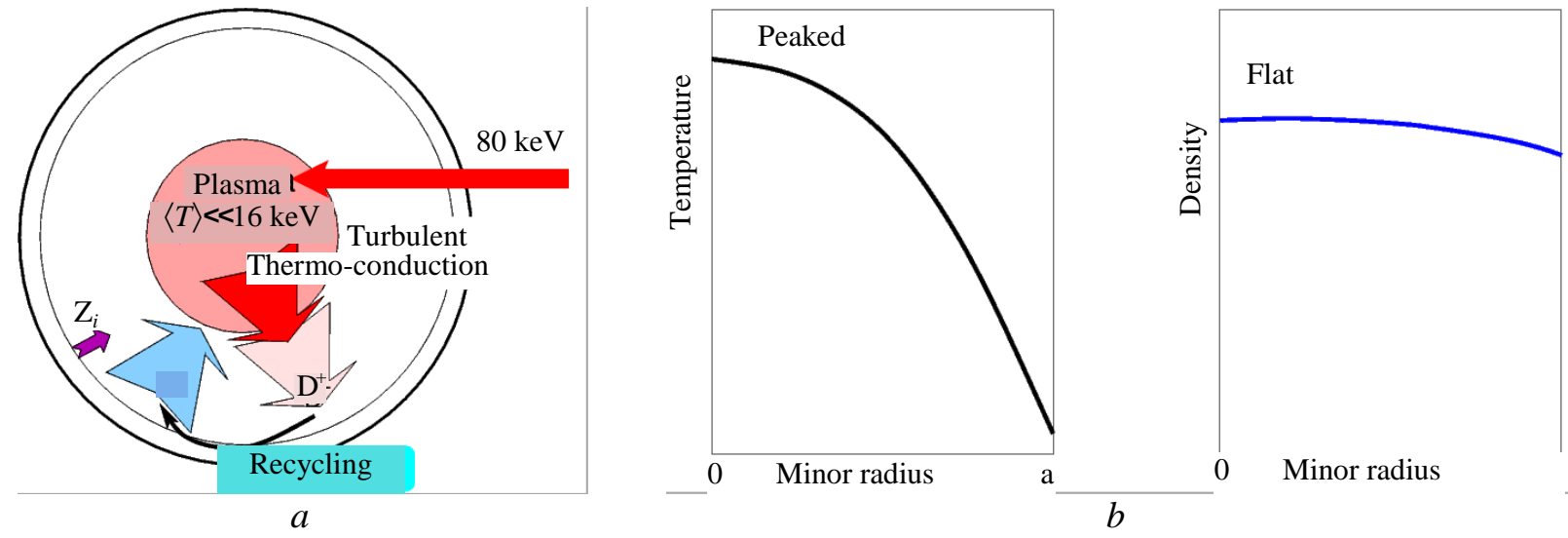

Fig. 1. (a) Conventional approach to magnetic fusion with the high recycling at the wall. $(b)$ The temperature profile is peaked, while the density profile is flat

The LiWF regime has much simpler physics as is shown in Fig. 2. The NBI delivers the particles and the energy right to the core. The recycling is highly suppressed, and the edge plasma temperature is as high as the core temperature. Moreover, it is directly related to the beam energy $E^{\mathrm{NBI}}$ as $T \approx E^{\mathrm{NBI}} / 5$.
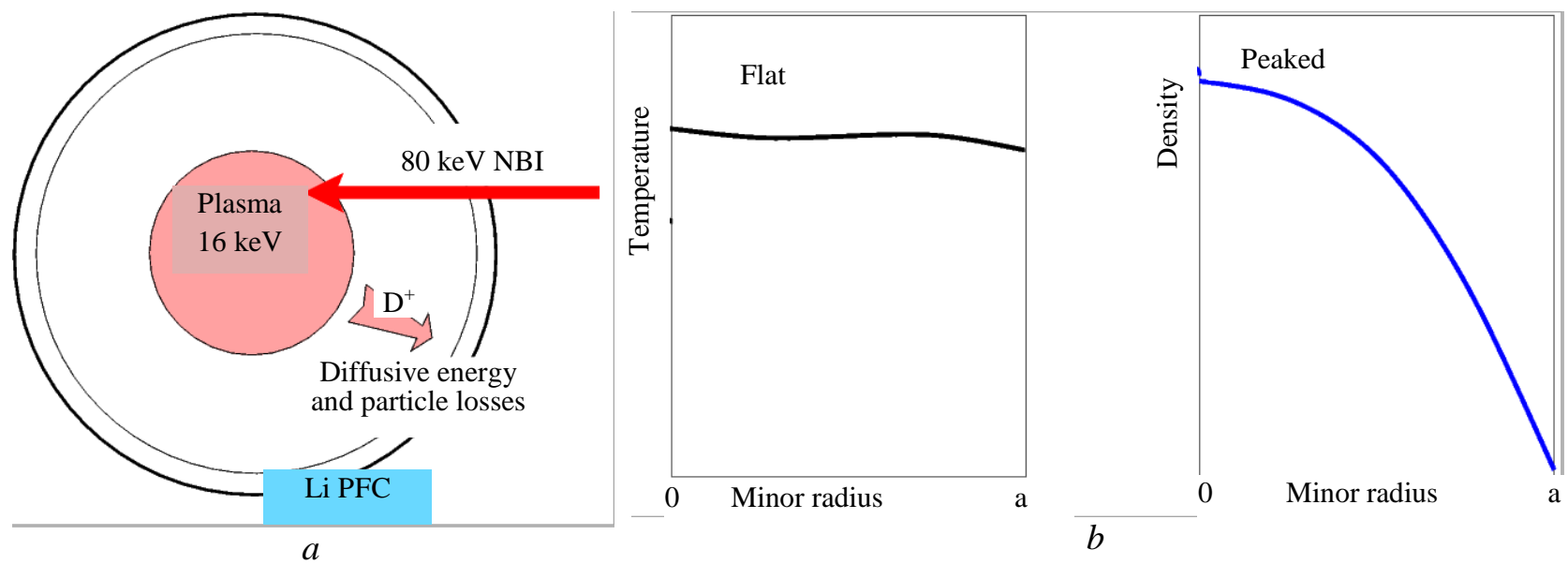

Fig. 2. (a) The LiWF regime with absorbing walls dramatically simplifies the physics. Energy goes to the wall with the core particle flux. (b) The temperature profile is flat, while the density profile is peaked

The practical implementation of the LiWF concept would lead to completely new plasma regimes. Everything is affected. The flat core temperature eliminates the temperature gradient related turbulence in the core. Moreover, the confinement becomes insensitive to any thermal conduction, both of ions and electrons. In the LiWF regime it is determined by the plasma diffusion. Unlike thermal conduction, which is unlimited due to turbulence, the diffusion is controlled by the best confined component, i.e., by ion diffusion. The anomalous thermal conduction of electrons, which up to now remains the major problem of conventional fusion, plays little or no role in the LiWF regime.

In the LiWF regime, the NBI controls the level of plasma temperature. The level of plasma density is determined by the NBI current and diffusion coefficient in the plasma, which is expected to be not larger than the ion neo-classical thermal conduction coefficient. Together with the bootstrap current, the deposition of the 
NBI controls the plasma current density in the non-inductive current drive regime.

Even in the inductive current drive regime, a flat electron temperature profile eliminates global core instabilities, such as sawtooth oscillations (which are also the trigger of the neo-classical tearing modes) and internal reconnection events in spherical tokamaks. The low plasma edge density (even with the high core density) eliminates the Greenwald density limit, thus, significantly improving the global plasma stability in the LiWF fusion regime. Remarkably, the Edge Localized Modes (ELMs), which are one of the major problem of the ITER project, are eliminated by stabilizing the near separatrix inner surfaces by the finite current density at the edge [7], or by reduction in the edge pressure gradient, or possibly by the reduction in the plasma edge density. In the LiWF regime, the Scrape Off Layer (SOL) becomes collisionless. This automatically eliminates the thermal force which may drive impurities from the divertor target plates toward the plasma surface and then, inside the plasma. The plasma interaction with the sidewalls, such as blobs, is not expected either. This makes the LiWF regime uniquely compatible with the stationary plasma regime, required for fusion-fission of pure fusion devices.

By no means, should the LiWF be considered simply as an improvement of the conventional approach to fusion, which not only did not resolve any of above mentioned problems, but, in fact, has enhanced their scale in large devices, including ITER. Also, the LiWF goes far beyond simple Li conditioning, which has shown impressive improvements of performance in existing experiments. The LiWF represents a new, self-consistent and promising concept of magnetic fusion. Five aspects of it are discussed in more details in the following sections.

\section{CORE FUELING AND LITHIUM REPLENISHMENT}

The Spherical Tokamaks (ST), like NSTX in PPPL suggest the easiest way to develop the LiWF regimes. Large plasma cross-section is convenient for testing different versions of the lithium covered target surfaces. ST is easy to fuel by NBI because of the small distance from the magnetic axis and surface of the plasma (on the low field side). At the same time the total thickness of the plasma is large enough for absorbing the entire beam.

Fig. 3, $a$ illustrates the core fueling of the ST plasma by NBI. Fig. 3, $b$ indicates that only divertor target surfaces should be covered by the lithium layer.

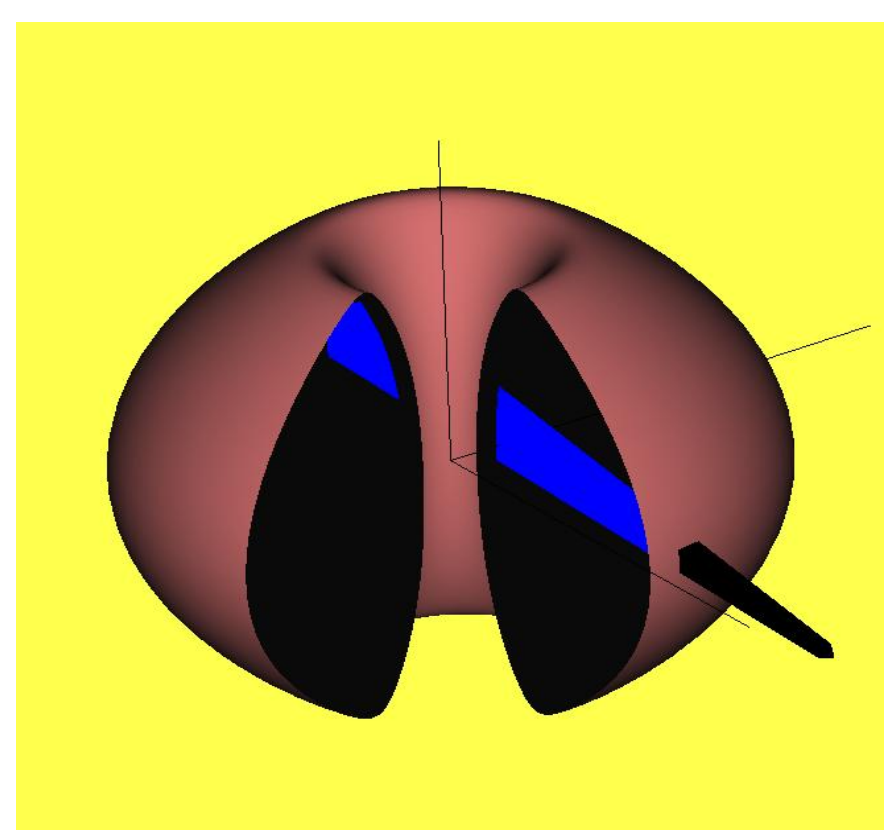

$a$

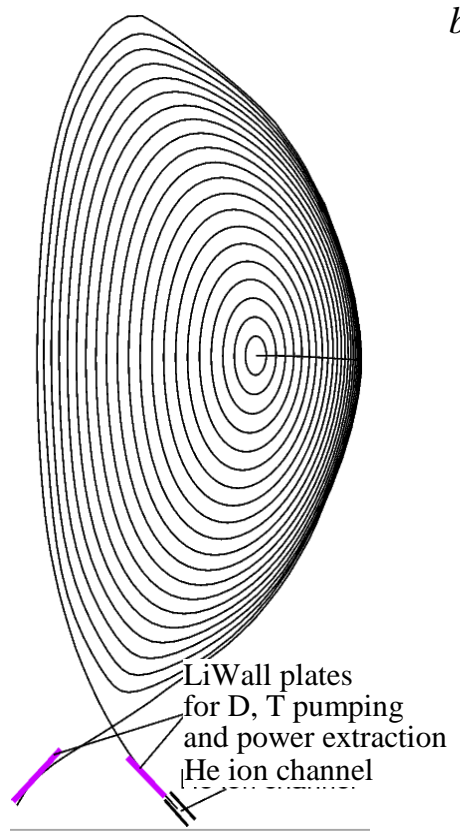

Fig. 3. Schematic implementation of LiWF in Spherical Tokamaks. (a) NBI fueling and heating the plasma.(b) Positioning the liquid lithium target plates in the divertor area for plasma particle pumping

As soon as the recycling is eliminated, the plasma temperature is directly determined by the beam energy $E^{\mathrm{NBI}}$. The beam particles are simply absorbed by the plasma and then are thermalized. As a result, the temperature is automatically flat (thermalization is much faster than the plasma diffusion) 


$$
E^{\mathrm{NBI}}=\frac{5}{2}\left(T_{i}+T_{e}\right), \frac{T_{i}+T_{e}}{2}=\frac{E^{\mathrm{NBI}}}{5} .
$$

In this relationship the coefficient $5 / 2$ consists of $3 / 2$, related to the definition of the temperature, and 1 , related to the diffusion of the plasma with a Maxwellian distribution function. Only this part of coefficient may depend on plasma properties. Otherwise, plasma physics is not present in the fundamental relationship (1).

For the typical beam energy $E^{\mathrm{NBI}}=80 \mathrm{keV}$, the plasma temperature in the ideal LiWF situation will be $16 \mathrm{keV}$. It is necessary to note, that the entire energy of NBI is in the ion component. As a result, the LiWF regime always corresponds to the "hot-ion" regime, which was proven to be the best one in tokamaks

$$
T_{i}>T_{e} \text {. }
$$

Unlike the temperature, the plasma density and its profile are determined by the NBI current, its deposition profile as well as by plasma diffusion. Without experiments it is not possible to predict the effect of diffusion. But even with such an uncertainty, the LiWF regime is much better controlled by external means, like NBI, than the conventional one with high recycling and turbulent plasma.

Concerning requirements for the lithium replenishment, in order to keep it capable of pumping the plasma particles, they are very modest. Thus, for NBI current of $50 \mathrm{~A}$ (4 MW for $80 \mathrm{keV}$ beam used in simulations) with 6 atomic \% of D-T concentration in lithium, the rate of Li replenishment is only $0.5 \mathrm{~g} / \mathrm{s}$. The natural speed $1 \mathrm{~cm} / \mathrm{s}$ under gravity of a $0.1 \mathrm{~mm}$ thick liquid lithium layer is sufficient practically in all possible cases. The real uncertainty is related to liquid lithium behavior under electro-magnetic forces from yet unknown currents from the plasma to the target plates. Only real experiment can show and resolve the possible issues, if any.

One of the issues which should be resolved in the future is the helium ash pumping from the fusion power producing devices. Lithium does not pump helium. The simplification is that helium ash from the plasma is released from the target plates with almost no energy. Without deeper discussion, the hopes are on the near double null plasma configuration. The inner separatrix can be used for diverting the core plasma particles to the lithium surface. On other hand, the outer separatrix can direct the low energy ionized helium to the cryopanel channels. Such a configuration will separate the energy extraction from the helium pumping by cryopumps.

\section{PLASMA EDGE TEMPERATURE}

In the present plasma, the edge particle source from the wall outgassing, gas puff and recycling is orders of magnitude larger than the NBI source. But even with the lithium plasma absorbing surfaces, the residual recycling and the gas flux to the plasma edge can be present. It will affect the general relationships outlined in the previous section.

Fig. 4 shows a simplified but realistic situation near the plasma edge. The plasma edge here is understood as the separation layer between the plasma core, which is the confinement zone, and the plasma periphery, where plasma flow and convection dominates over diffusive transport.

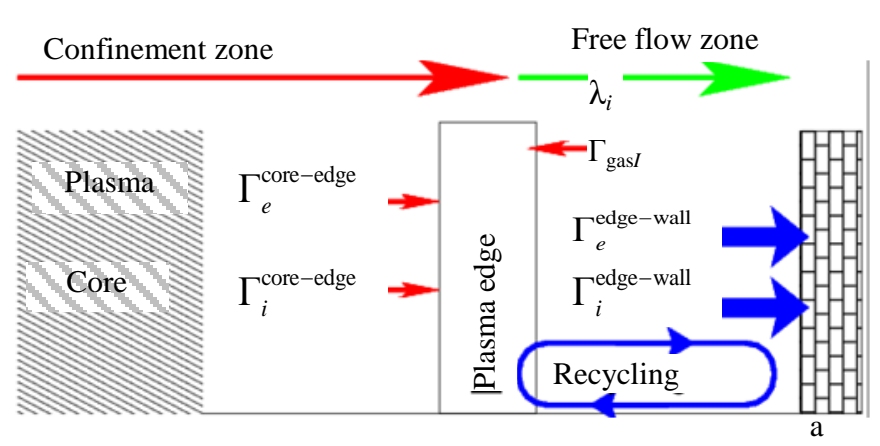

Fig. 4. Plasma edge, separating the core confinement zone and the "free" flow zone, and the plasma particle fluxes to the edge from the core side and from the wall side (including recycling and gas injection)
In a highly collisional SOL, such an edge is localized near the wall at the distance of the mean free path $\lambda_{\|}$ along the magnetic field. For deuterium ions it is

$\lambda_{\|, \mathrm{D}}[\mathrm{m}] \cong 121 \frac{T^{2}[\mathrm{keV}]}{n_{20}}$,

where $n_{20}$ is the edge plasma density in $10^{20} \mathrm{~m}^{-3}$ units. The question is the location of the plasma edge for the high edge temperature $T[\mathrm{keV}]>1$, when $\lambda_{\|}$is approaching $10^{3} \mathrm{~m}$ and exceeds the connection lengths in the SOL. The simple answer that the edge is situated at the separatrix appears to be incorrect. The fluxes $\Gamma_{i}^{\text {core-edge }}, \Gamma_{e}^{\text {core-edge }}$ from the core and from the gas injection $\Gamma^{\text {gas } I}$ are amplified by recycling at the walls. The resulting particle fluxes to the wall are given by 


$$
\begin{gathered}
\Gamma_{i}^{\text {edge-wall }}=\frac{\Gamma_{i}^{\text {core-edge }}+\Gamma^{\text {gas } l}}{1-R_{i}}, \\
\Gamma_{e}^{\text {edge-wall }}=\frac{\Gamma_{e}^{\text {core-edge }}+\Gamma^{\text {gas } l}}{1-R_{e}},
\end{gathered}
$$

where $R_{\mathrm{i}}$ and $R_{e}$ are the recycling coefficients for the ions and electrons correspondingly. Neglecting radiation, the energy to the wall is conveyed exclusively by the particle flux

$$
\begin{aligned}
& \frac{5}{2} \Gamma_{i}^{\text {edge-wall }} T_{i}^{\text {edge }}=\int_{V} P_{i} \mathrm{~d} V-\frac{\partial}{\partial t} \int_{V} \frac{3}{2} n T_{i} d V, \\
& \frac{5}{2} \Gamma_{e}^{\text {edge-wall }} T_{e}^{\text {edge }}=\int_{V} P_{i} \mathrm{~d} V-\frac{\partial}{\partial t} \int_{V} \frac{3}{2} n T_{e} d V,
\end{aligned}
$$

where $T_{i}$ and $T_{e}$ are ion and electron temperatures $P_{i}$ and $P_{e}$ are the core heating sources for the ions and electrons correspondingly and the integration is over the plasma volume. Assuming the core fueling from the NBI

$$
\Gamma_{i}^{\mathrm{core}-\text { edge }}=\Gamma_{e}^{\mathrm{core}-\mathrm{edge}}=\Gamma^{\mathrm{NBI}} .
$$

The above two sets of equations can be combined into a boundary condition for the edge temperature

$$
\frac{T_{i}^{\text {edge }}+T_{e}^{\text {edge }}}{2} \geq \frac{1-R_{e i}}{1+\Gamma^{\text {gasI }} / \Gamma^{\mathrm{NBI}}} \cdot \frac{E^{\mathrm{NBI}}+E^{\mathrm{aux}}}{5},
$$

where

$$
R_{e i} \equiv \max \left\{R_{e}, R_{i}\right] .
$$

The effective energy $E^{\text {aux }}$ of the additional to NBI heating power source $P^{\text {aux }}$ is defined by

$$
E^{\mathrm{aux}} \equiv \frac{P^{\mathrm{aux}}}{I^{\mathrm{NBI}}}
$$

where $I^{\mathrm{NBI}}$ is the $\mathrm{NBI}$ current.

The equation (9) indicates that in order to make the edge temperature comparable to the reference value $E^{\mathrm{NBI}} / 5$ the recycling coefficient $R_{e i}$ should be less than at least $50 \%$ and the gas flux to the plasma surface should be smaller than the beam particle source.

The similar consideration leads to the plasma edge density $n^{\text {edge }}$ in the form

$$
n^{\text {edge }} \approx \frac{\left\langle n^{\text {core }}\right\rangle}{\left(1-R_{e i}\right)} \cdot\left(1+\frac{\Gamma^{\text {gas } I}}{\Gamma^{\mathrm{NBI}}}\right) \cdot \frac{\delta}{a} .
$$

This formula is approximate and expressed in terms of the volume averaged plasma core density $n^{\text {core }}$, minor radius $a$ and a characteristic scale $\delta$ (such as banana width) determining the unidirectional ion flux from the core to the edge. In the LiWF regime $n^{\text {edge }}$ is much smaller than $\left\langle n^{\text {core }}\right\rangle$ (average density in the core).

The thing of exceptional importance is that even with residual effects of recycling or the gas flux, the high plasma edge temperature is in full control by external means, i.e. heating power and wall conditions. It does not depend on the core plasma properties. In the LiWF regime the plasma edge temperature is under external control.

In its turn the edge controls the core. Numerous uncertainties, related to the turbulent thermal conduction in the presently dominant approach to fusion, are eliminated in the LiWF concept.

In Eq. (9) the edge temperature is a boundary condition, independent of the local transport coefficient. This simple fact explains the DIII-D experiments with Resonant Magnetic Perturbations, when the pedestal electron temperature was not affected by the local destruction of magnetic configuration. The experiments are consistent with understanding of the plasma edge, given by the LiWF theory, which predicts the edge location at the top of the temperature pedestal, rather than at the separatrix as is widely assumed. The LiWF theory has also dismissed the notion of the "edge transport barrier" as a misconception. The pedestal region is located outside the confinement zone and is full of magnetic stochasticity. 


\section{DIFFUSION BASED CONFINEMENT}

The Eqs. $(9,12)$ specify the boundary conditions for the core, where the transport of the energy and particles is diffusive (i.e., related to the gradients of plasma profiles).

In STs (e.g., NSTX), even in the presence of turbulence the ions behave neo-classically [8]. A similar situation is frequent even in conventional tokamaks.

Because in the LiWF regime the ions will determine the losses, irrespective to the electron anomaly, the reasonable transport simulation model for the LiWF is

$$
D=\chi_{\mathrm{i}}=\chi_{i}^{\text {neo }}, \chi_{e}=f \chi_{i}^{\text {neo }}, \quad 1 \leq f \leq \infty .
$$

Here $D$ is the coefficient of particle diffusion, $\chi_{i}$ and $\chi_{e}$ are the ion and electron thermal conduction coefficients determining the particle flux $\Gamma_{i, e}^{\text {core }}$ and the heat fluxes $q_{i}^{\text {core }}$ and $q_{e}^{\text {core }}$ in the ion and electron channels:

$$
\begin{gathered}
\Gamma_{i, e}^{\text {core }}=-D \nabla n, \\
q_{i}^{\text {core }}=-n \chi_{i} \nabla T_{i}, \quad q_{e}^{\text {core }}=-n \chi_{e} \nabla T_{e} .
\end{gathered}
$$

The factor $f$ is introduced here to simulate the effect of the anomaly of electron thermal conductivity. In the case of $f=1$ the above transport model is called Reference Transport Model (RTM). As a reference, it does not need deep justification. But it is consistent with the present data concerning neo-classical ions. At the same time, RTM is not a neo-classical model. Its diffusion coefficient is almost 60 times larger than the neo-classical value. The RTM with the LiWF boundary conditions has been implemented in the ASTRA-ESC transport-equilibrium code system [9]. The model was tested against the CDX-U data [10, 11]. Because of the relatively long mean free path of cold neutrals in the low density CDX-U plasma, the particle source was calculated by solving the kinetic equation for a neutral distribution function describing multiple charge exchange and ionization.

Comparison of RTM with CDX-U data

\begin{tabular}{l|c|c|c|c|c}
\hline \multicolumn{1}{c|}{ Parameter } & CDX-U & RTM & RTM-0.8 & RTM-0.65 & glf23 \\
\hline Fueling ${ }^{*}, 10^{21} / \mathrm{sec}$ & $1-2$ & 1 & 0.5 & 0.3 & $0.8-3$ \\
$\beta_{j}$ & 0.150 & 0.151 & 0.150 & 0.151 & 0.145 \\
$l_{i}$ & 0.66 & 0.769 & 0.702 & 0.671 & 0.877 \\
$V$, Volt & $0.5-0.6$ & 0.77 & 0.53 & 0.40 & 0.85 \\
$\tau_{E}, \mathrm{msec}$ & $3.5-4.5$ & 2.7 & 3.8 & 5.3 & 2.3 \\
$n_{e, 20}(0)$ & - & 0.09 & 0.07 & 0.0590 & 0.09 \\
$T_{e}(0), \mathrm{keV}$ & - & 0.308 & 0.366 & 0.413 & 0.329 \\
$T_{i}(0), \mathrm{keV}$ & - & 0.031 & 0.029 & 0.030 & 0.028 \\
${ }^{*}$ Adjusted to econstructed $\beta_{j}$ & & & & & \\
\hline
\end{tabular}

The table correspond to CDX-U measurements (or equilibrium reconstruction results), the RTM with the diffusion coefficients equal to $100 \%, 80 \%, 70 \%$ and $65 \%$ of its canonical RTM value (13). The last column corresponds to the turbulent glf 23 transport model, used in conventional fusion simulations.

In simulations, only the gas puff value was adjusted to the measured value (ratio of the thermal and poloidal magnetic energy) of the plasma. The RTM, especially RTM- 0.8 , has easily reproduced three plasma parameters: the loop voltage, internal inductance, and the energy confinement time extracted using the equilibrium reconstruction. At the same time it was impossible to adjust the gas puff value for the glf23 model in order to match the same number of parameters.

For illustration of the effect of finite recycling and anomalous electron thermal conduction, the ASTRA-ESC calculations have been performed for a particular spherical nokamaks, called ST1, which the author is promoting as a candidate for the next step machine in PPPL.

Basic parameters of ST1:

$\begin{array}{ll}R_{\max }, \mathrm{m} \ldots & 1.68 \\ R_{0}, \mathrm{~m} \ldots & 1.05 \\ a, \mathrm{~m}, \ldots & 0.63 \\ B, \mathrm{~T} \ldots & 1.5\end{array}$




$\begin{array}{ll}\beta, \ldots & 0.2 \\ I_{\mathrm{pl}}, \mathrm{MA} \ldots & 4 \\ P^{\mathrm{NBI}}, \mathrm{MW} \ldots & 1-3 \\ E^{\mathrm{NBI}}, \mathrm{keV} \ldots & 80 \\ P_{\mathrm{DT}}^{\text {equiv }} \ldots & 10-20 \\ Q_{\mathrm{DT}}^{\text {equiv }} \ldots & 5-8\end{array}$

The plots of the energy confinement time in ST1versus electron anomaly factor $f$, are presented in Fig. 5.

The horizontal line in Fig. 5 for the no recycling case, $R_{e i}$ $=0$, illustrates the complete independence of the energy confinement time from any thermal conduction. The energy confinement time is simply equal to $3 / 5$ of the particle confinement time in the core.

The outstanding result is that even for finite recycling up to 0.5 there is practically no dependence of energy confinement on the electron thermal conduction.

Such a modest and practically achievable level of recycling suppression by the lithium surface would resolve a number of fundamental problems of conventional magnetic fusion.

\section{CONFINEMENT AND THE POWER EXTRACTION PROBLEM}

The confinement expected from the RTM model is so good that the NBI is perfectly sufficient for fueling. In the above example, it requires only $1-3 \mathrm{MW}$ (depending on recycling) of the NBI power in order to get in ST1 10-20 MW of the fusion power (similar to what was achieved on JET or TFTR with much higher heating power).

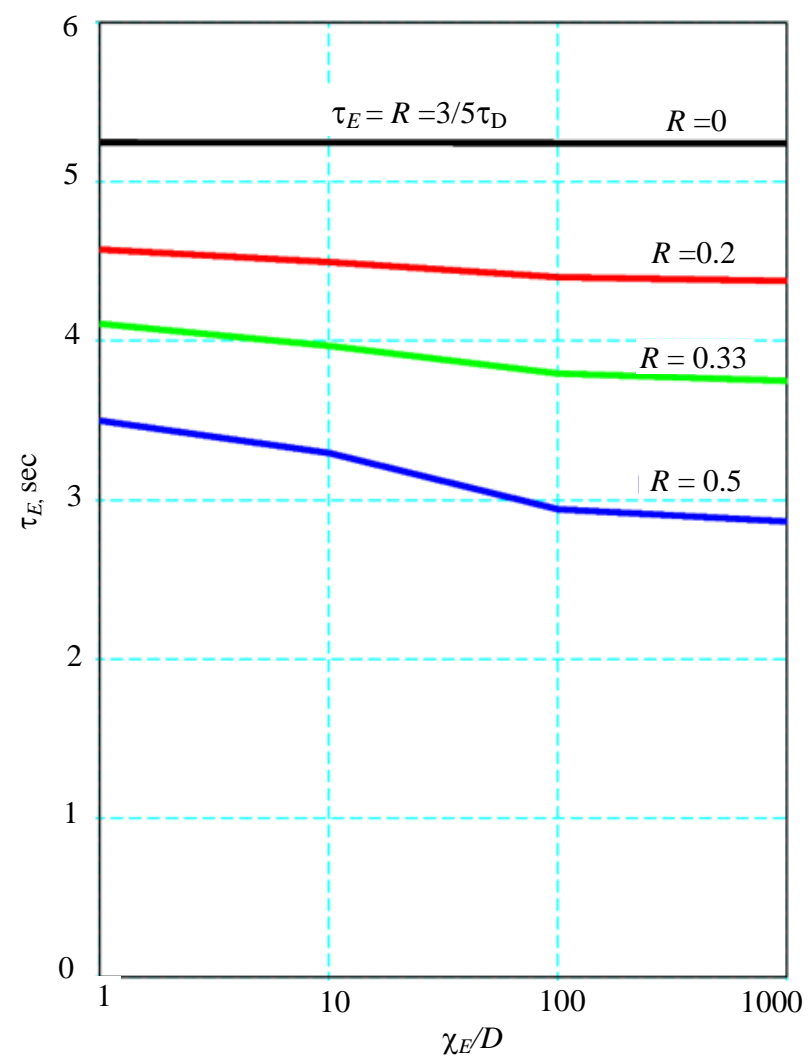

Fig. 5. Energy confinement time for ST1 device as a function of anomaly in the electron thermal conductivity for different recycling coefficients (gas injection is absent)

The fundamental conclusion is that the LiWF regime does not need $\alpha$-particle assistance in heating the plasma. This removes a big set of problems related to $\alpha$-particles. In particular, it is not required for ST to confine $\alpha$ particles, which would need very high plasma currents (more than $10 \mathrm{MA}$, highly problematic for STs).

In the case of a conventional tokamak, e.g., for the fusion-fission purposes, where the plasma current could easily be sufficient for $\alpha$-particles confinement, the magnetic field can be adjusted in order to irradiate by cyclotron radiation the extra electron energy obtained from the $\alpha$-particles. In both cases the $\alpha$-particles energy does not go to the divertor target plates.

By enhancing confinement and reducing the plasma heating power, the LiWF suggests a practical solution to the power extraction problem, which remains unsolved within the conventional approach.

But even in terms of power extraction itself, the lithium based target plates are advantageous with respect to conventional, high- $\mathrm{Z}$ ones. The power extraction abilities of the plates are determined by the coolant side of the channels in the plates rather than by the plasma facing surface. The sandwich of the thin liquid layer of lithium on the interface thin layer of stainless steel brazed to the copper heat sink, allows the use of copper for the heat removal. As a reference, the $1 \mathrm{~cm}$ thick copper plate with the coolant temperature of $150{ }^{\circ} \mathrm{C}$ and the Li surface temperature of $300{ }^{\circ} \mathrm{C}$ can extract at least $4.5 \mathrm{MW} / \mathrm{m}^{2}$ heat flux.

Even with limitations on the lithium surface temperature such a sandwich would allow conducting the same heat flux from the plasma as for conventional plate designs.

On the other hand, from the plasma physics point of view, the situation is much better in the case of the LiWF, where ELMs are eliminated, while for conventional fusion they represent the outstanding problem due to plate erosion. 


\section{LiWF BASED NEUTRON SOURCES}

The development of the LiWF regime on the existing NSTX device would be a real step to practical fusion, which desperately requires a powerful neutron source for testing materials and providing data for the reactor design or for fusion-fission applications.

Spherical tokamaks, still having the mission in demonstrating discharge initiation and stationary current drive, represent the only fast track option for such a neutron source, called here the Reactor Development Facility (RDF). Based on RTM simulations the cross-sections of three ST steps toward RDF are presented in Fig. 6.

Here, ST0 is the NSTX device, adjusted for development of the LiWF regime by installation of the lithium covered target plates in the lower divertor. Besides the technological and operational challenges, its mission is to demonstrate the three-four fold jump in the energy confinement time (up to $250-300 \mathrm{~ms}$ ).

The next ST1 device can target the condition

$$
\langle p\rangle \tau_{E}=1[\mathrm{MPa} \cdot \mathrm{s}],
$$

( $\langle p\rangle$ is the volume averaged plasma pressure, $\tau_{E}$ is the energy confinement time). In the case of the $\alpha$-particle confinement, this would be the ignition condition.

ST1 would be the first machine specifically designed for the LiWF regime. It should provide the data for designing the ST2, which is the DD-prototype of RDF. ST2 should develop most of plasma physics aspects (plasma control, power extraction, He pumping, current drive, etc) of the burning plasma regime for RDF using the DD-plasma.

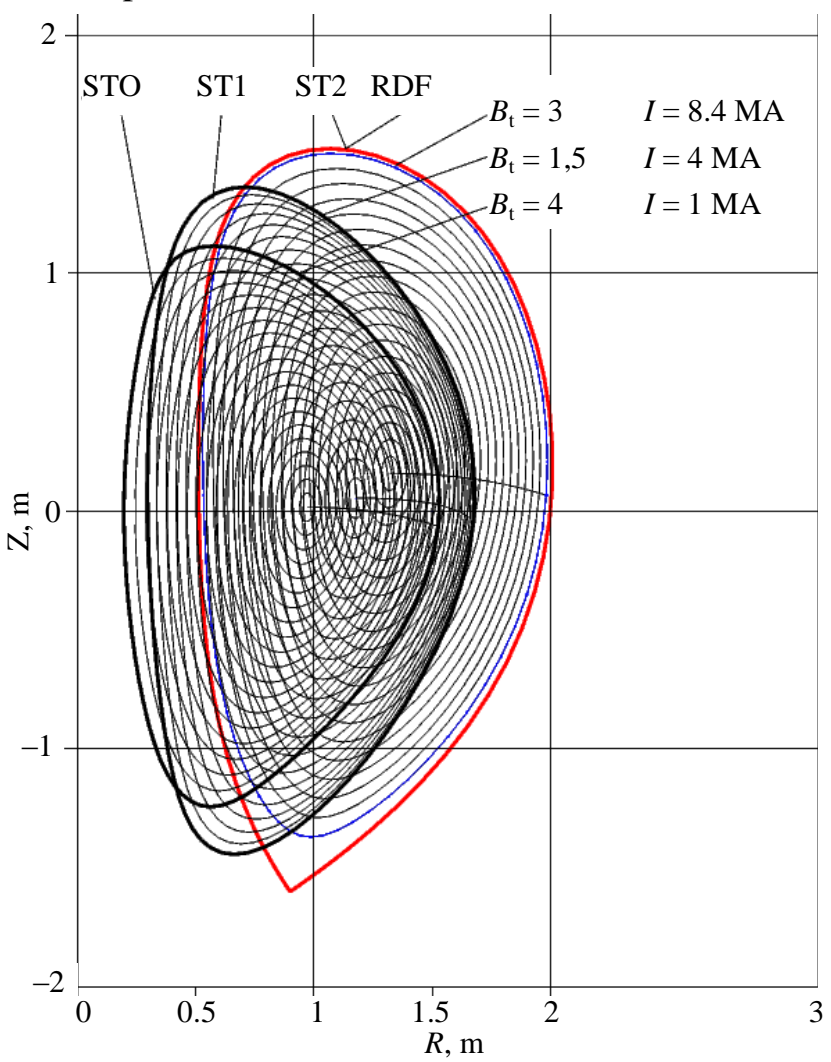

Fig. 6. Plasma cross-sections of NSTX (ST0), ST1, and identical ST2 and RDF
In this strategy, ST2 will leave for RDF only the issues related to tritium, energetic $\alpha$-particles (including power extraction and additional plasma heating), testing materials and reactor components at the fusion power of $0.2-0.5 \mathrm{GW}$.

Fig. 7 gives the comparison between the LiWF path of development with the ITER step. $a$

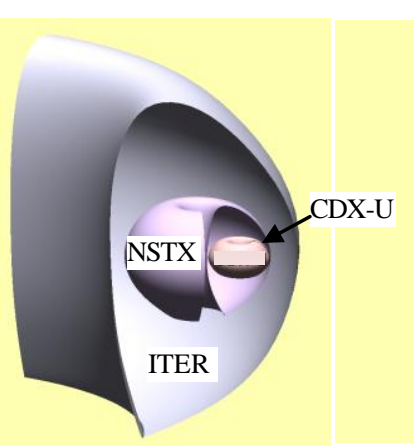

$b$

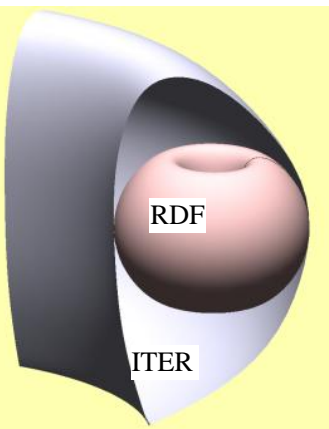

Fig. 7. Plasma configuration of (a) CDX-U and NSTX, experimental facilities for LiWF studies, and $(b)$ the $0.2-0.5 \mathrm{GW}$ Reactor Development Facility, considered as a feasible goal for the LiWF approach 


\section{CONCLUSION}

The LiWF concept, simple, consistent with the relevant experiments, successful in prediction of enhancement of the energy confinement time, flattening the temperature profile, elimination of internal core MHD instabilities, elimination of ELMs, successful in understanding the plasma edge and in dismantling the long standing misconception of the edge transport barrier, successful in many other aspects, is still only at the very beginning of penetration into the experimental programs.

Curiously, the success with lithium conditioning, which is a step to the LiWF regimes, in certain aspects is distracting the fusion program from the necessary development of the liquid lithium related technology.

But the results from different machines now are encouraging and convincing for a growing number of people. It is necessary to recognize that the switch to the LiWF fusion is unavoidable. With a few yet conceptually unclear issues (such as helium pumping), the concept is uniquely self-consistent, relies on a very basic plasma physics and promises the fast progress in fusion development.

This work is supported by US DoE contract No. DE-AC02-09-CH11466.

\section{REFERENCES}

1. McGuire, K.M. et al. — Fusion Energy, 1996 (Proc. 16th Intern. F.E. Conf. Montreal, IAEA), Vienna, 1997, vol. 1, p. 19.

2. Keilhacker M. et al. - Nucl. Fusion, 1999, vol. 39, p. 209.

3. Evtikhin, V.A. et al. - Fusion Eng. Des., 2001, p. 363.

4. Krasheninnikov S.I. et al. - Phys. of Plasmas, 2003, vol. 10, p. 1678.

5. Zakharov L.E. et al. — Fusion Eng. Des., 2004, vol. 72, p. 149.

6. http://w3.pppl.gov/ zakharov, professional web-site "Lithium: the key to fusion power".

7. Zakharov L.E. et al. - J. Nucl. Mater., 2007, vol. 363-365, p. 453.

8. Kaye S.M. et al. - Phys. Rev. Letters, 2007, vol. 98, p. 175002.

9. Pereverzev G.V. et al. ASTRA-Automated system for transport analysis in a tokamak. IPP Garching Report IPP5/98 February 2002.

10.Majeski R. et al. — Phys. Rev. Lett., 2006, vol. 97, p. 075002.

11.Majeski R. et al. - Nucl. Fusion, 2009, vol. 49, p. 055014.

\section{AUTHORS}

Zakharov L.E. Princeton University, Princeton Plasma Physics Laboratory, MS-27, P.O. Box 451, Princeton, New Jersey 08543; zakharov@ pppl.gov.

Received 19 June 2010

Problems of Atomic Science and Technology Ser. Thermonuclear Fusion, 2011, issue 1, pp. 29-38 